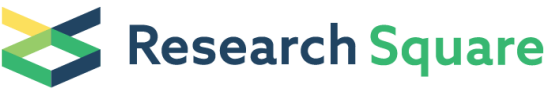 \\ Preprints are preliminary reports that have not undergone peer review. \\ They should not be considered conclusive, used to inform clinical practice, or referenced by the media as validated information.
}

\section{Preparation of Cellulose-based fluorescent materials as coating pigment by use of reversible DMSO/DBU/CO2 system}

\section{Qinghua Cao}

Hainan University

Jinyue Dai

Ningbo Institute of Materials Technology and Engineering CAS: Ningbo Institute of Industrial Technology Chinese Academy of Sciences

\section{Xin Bao}

Ningbo Institute of Materials Technology and Engineering CAS: Ningbo Institute of Industrial Technology Chinese Academy of Sciences

\section{Zhenyu Zhang}

Ningbo Institute of Materials Technology and Engineering CAS: Ningbo Institute of Industrial Technology Chinese Academy of Sciences

\section{Fei Liu}

Ningbo Institute of Materials Technology and Engineering CAS: Ningbo Institute of Industrial Technology Chinese Academy of Sciences

\section{Yuhong Feng}

Hainan University

\section{Haining Na ( $\nabla$ nahaining@nimte.ac.cn )}

Ningbo Institute of Materials Technology and Engineering, Chinese Academy of Sciences https://orcid.org/0000-0001-8067-9962

\section{Jin Zhu}

Ningbo Institute of Materials Technology and Engineering CAS: Ningbo Institute of Industrial Technology Chinese Academy of Sciences

\section{Research Article}

Keywords: Cellulose, Reversible DMSO/DBU/CO2 system, Fluorescent material

Posted Date: May 10th, 2021

DOI: https://doi.org/10.21203/rs.3.rs-218340/v2

License: @ (i) This work is licensed under a Creative Commons Attribution 4.0 International License. Read Full License 


\section{Abstract}

A series of cellulose-based fluorescent materials are prepared under relative mild conditions by use of the reversible DMSO/DBU/ $/ \mathrm{CO}_{2}$ system to utilize as coating pigments. Through the observation under $365 \mathrm{~nm} \mathrm{UV}$ light, the cellulose-based fluorescent materials exhibit good fluorescence response and bright color. Furthermore, due to the limitation of the molecular skeleton of cellulose, the intrinsic aggregation caused quenching phenomenon commonly existed in conventional organic fluorescent pigments can be effectively inhibited, which is very helpful to retain good fluorescence response in epoxy-based coating material and its coating films. Moreover, the addition of cellulose-based fluorescent materials also increases the mechanical properties of the coating film. The increase of tensile strength and tensile modulus respectively reaches 39\% and $\sim 66 \%$. Solvent resistance and thermal property of the coating films generally remain unchanged. The fabrication of cellulose-based fluorescent materials in $\mathrm{DMSO} / \mathrm{DBU} / \mathrm{CO}_{2}$ system provides a feasible way to develop the functional application of cellulose.

\section{Introduction}

With the constant exhaustion of petrochemical energy and the aggravation of environmental pollution, the development and utilization of clean and renewable bioresources have been widely attended by people (Llevot et al., 2016; Teng et al., 2010). As one of the most important green bioresources with good performance, cellulose is strongly expected to be used as renewable material (Druel et al., 2018). But, in general, cellulose has very strong intermolecular and intramolecular hydrogen bonds. It makes cellulose difficult to processing and forming (H. Zhang et al., 2005; L. Zhang et al., 2019). This defect weakens the applicability of cellulose.

By use of functional modification (Heinze \& Liebert, 2001; Klemm et al., 2005; Wu et al., 2004; Zheng et al., 2005), to convert cellulose into ether or ester derivatives, the processing ability and potential application are definitely improved. Usually, the functionalization of cellulose could be carried out via heterogeneous and homogeneous approaches (Klemm et al., 2005). The preparation of cellulose derivative in heterogeneous nondissolving system used to be the mainstream approach and mainly for industrial products (Heinze \& Liebert, 2001). However, by using heterogeneous system, the effect and efficiency of functional modification is commonly at low level (Wu et al., 2004). In order to establish a homogeneous system to increase ability and quality of cellulose functionalization, during past decades, many dissolving systems have been developed such as N,N-dimethylacetamide/LiCl (DMAc/LiCl) (Mccormick \& Dawsey, 1990), N-methylmorpholine- $\mathrm{N}$ oxidemonohydrate (NMMO) (Fink et al., 2001), DMSO/Tetrabutylammoniumfluoride (DMSO/TBAF) (Koehler \& Heinze, 2007), molten salt hydrates $\left(\mathrm{LiClO}_{4} \cdot 3 \mathrm{H}_{2} \mathrm{O}\right)$ (Fischer et al., 2003), $\mathrm{NaOH} /$ water/urea (or thiourea) solutions (Zhou \& Zhang, 2000) and ionic liquids (ILs) (Andanson et al., 2014; Canche-Escamilla et al., 2002). With the help of these dissolving systems, the more efficiently functional modification of cellulose can be achieved. In recent years, a reversible DMSO/organic base/ $\mathrm{CO}_{2}$ system has been further developed to dissolve cellulose (Q. Zhang et al., 2013). In this system, the hydroxyl of cellulose firstly reacts with amine base and $\mathrm{CO}_{2}$ to produce a kind of temporary carbonate structure, which forms similar effect of ionic liquid. This process can activates the hydroxyl of cellulose and promotes the solubility of microcrystalline cellulose (Xie et al., 2014). This method not only produces a homogeneous dissolution system, but also improves the modification ability of cellulose even under mild reaction condition, such as the acylation with anhydride 
(Yang et al., 2015), grafting with polylactide (Song et al., 2015) and polyester (Xu et al., 2017), and substitution with halides (Onwukamike et al., 2017). Though many researches have involved the modification of cellulose, few reports mentioned the functional cellulose derivatives. Furthermore, the fluorescent cellulose derivative obtained by reversible DMSO/DBU/CO ${ }_{2}$ system has not been reported. In fact, the fluorescent derivative modification of cellulose is an important way to create cellulose-based fluorescent materials which promote the application of this green bioresource material. Furthermore, common fluorescent pigments usually have advantages as diverse structure, bright color and strong tinting strength. But as relatively small molecular chemicals, they tend to migrate and to reunite (Rahman et al., 2009). This phenomenon leads to the wellknown aggregation caused quenching (ACQ) (Birks, 1971). As a result, the actual color of the common fluorescent pigments will be much dim. In contrast, the cellulose-based fluorescent materials can be easily controlled with stable chemical structure to achieve good solvent resistance and chemical stability. More notably, it can inhibit the unnecessary migration of fluorescent groups and possibly avoid the ACQ to retain bright color.

In this work, cellulose was utilized to dissolve in the reversible DMSO/DBU/ $\mathrm{CO}_{2}$ system to prepare cellulosebased fluorescent materials. And four kinds of fluorescent groups were respectively introduced into cellulose derivatives. The fluorescent response of the cellulose-based fluorescent materials was researched. After that, these fluorescent cellulose derivatives were further tried to add into epoxy resin as pigments to fabricate the thermosetting fluorescent coatings. Fluorescent response and mechanical properties of the fluorescent coatings were also discussed in the experiments.

\section{Materials And Methods}

\subsection{Materials}

Corncob cellulose was obtained from Shandong Shengquan Co.,Ltd. Microcrystalline cellulose (MCC) was purchased from Jiangsu LongHao new materials company. $\mathrm{CO}_{2}$ with a purity of $>99.99 \%$ was supplied from Ningbo Wanli Gas Company. DMSO (>99\%), 1,8-diazabicyclo[5.4.0]undec-7-ene (DBU), diethylenetriamine (DETA), KH560 silane coupling agent, 2-(bromomethyl)naphthalene (2-BMN), 9-(chloromethyl) anthracene (9CMA), coumarin 151 and 1-(bromomethyl)pyrene (1-BMP) were all obtained from Aladdin Commerce Reagent Co. Ltd. Waterborne epoxy resin (CYDW-100, epoxy value about 0.51) was obtained from Zhengzhou Penghui Company. All the chemical reagents are used without additional treatments.

\subsection{Dissolving cellulose in $\mathrm{DMSO} / \mathrm{DBU} / \mathrm{CO}_{2}$ system and synthesis of cellulose-based fluorescent materials}

Cellulose $(0.235 \mathrm{~g})$, DMSO $(10 \mathrm{~g}, 128 \mathrm{mmol})$ and DBU $(0.75 \mathrm{~g}, 4.93 \mathrm{mmol})$ were added into a high-pressure reactor, and then kept stirring while continuously pumping $\mathrm{CO}_{2}$ (5bar) for $2 \mathrm{~h}$ at $50^{\circ} \mathrm{C}$ to obtain clear and transparent cellulose solution ( $2 \mathrm{wt} \%$ ). The cellulose solution was kept at $50^{\circ} \mathrm{C}$ in the reactor for $1 \mathrm{~h}$ and then cooled to room temperature. Then 2-BMN $(0.32 \mathrm{~g}, 1.45 \mathrm{mmol})$ was carefully added to the obtained cellulose solution and then followed with a vigorous mechanical stirring for $4 \mathrm{~h}$ at $30^{\circ} \mathrm{C}$. After finishing the derivative reaction, the mixture was poured into methanol $(100 \mathrm{~mL})$ and washed twice continuously with distilled water $(50 \mathrm{~mL})$ and methanol $(50 \mathrm{~mL})$, respectively. The precipitate was dried at $80^{\circ} \mathrm{C}$ for $24 \mathrm{~h}$ to obtain powder as final product A. The synthesis processes of the other cellulose-based fluorescent materials were exactly the same. 
The cellulose-based fluorescent materials produced with Coumarin 151 (0.332g, 1.45mmol), 1-BMP (0.428g, $1.45 \mathrm{mmol})$, and $9-\mathrm{CMA}(0.328 \mathrm{~g}, 1.45 \mathrm{mmol})$ are respectively marked with $\mathrm{B}, \mathrm{C}$ and $\mathrm{D}$. The schematic process to synthesize cellulose-based fluorescent materials is shown in Figure 1.

\subsection{Preparation of epoxy coating films}

The cellulose and cellulose-based fluorescent materials (with particle diameter $\sim 20 \mu \mathrm{m}$ ) were immersed in $2 \mathrm{wt} \% \mathrm{NaOH}$ aqueous solution $(500 \mathrm{~mL})$, respectively. The container was placed in a magnetic stirrer for $4 \mathrm{~h}$ at $60^{\circ} \mathrm{C}$ until the reaction was completed. Alkali treatment, the powders were filtrated and washed several times

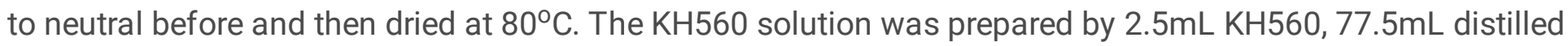
water and $120 \mathrm{~mL}$ anhydrous ethanol with stirring continuously for $2 \mathrm{~h}$. Then cellulose and cellulose-based fluorescent materials were added into $\mathrm{KH} 560$ solution for $4 \mathrm{~h}$ to fully coupling and then dried at $80^{\circ} \mathrm{C}$ for further use. $0.5 \mathrm{~g}$ of cellulose and cellulose-based fluorescent materials were dispersed in $12 \mathrm{~mL}$ of water and treated with ultrasound for $4 \mathrm{~h}$.

Epoxy resins $(10 \mathrm{~g})$ and curing agent (DETA, $1.3 \mathrm{~g})$ in the stoichiometric ratio (epoxy group/ $\mathrm{N}-\mathrm{H}=1: 1$ ) were wellmixed in a beaker. After that, cellulose-based fluorescent materials $(0.5 \mathrm{~g})$ were added with stirring continuously for $10 \mathrm{~min}$. Then the mixtures were quickly transferred to a stainless mold. The thermal curing reaction was performed on a heater at $80^{\circ} \mathrm{C}$ for $3 \mathrm{~h}$. Finally, the mold was slowly cooled down to room temperature. The cured epoxy resin films (thickness $200 \mu \mathrm{m}$ ) were removed from the mold and then left at room temperature for $24 \mathrm{~h}$. The epoxy coating film with cellulose $(0.5 \mathrm{~g})$ was also prepared as blank sample.

\subsection{Characterization}

The FT-IR analysis of the synthesized cellulose-based fluorescent materials was performed on a NICOLET $6700 \mathrm{FT}-\mathrm{IR}$ using the $\mathrm{KBr}$ pellet method with transmittance mode. The spectra recorded from 400 to $4000 \mathrm{~cm}^{-1}$ at room temperature and 16 scans were collected for each sample. The ${ }^{1} \mathrm{H}-\mathrm{NMR}$ spectra were recorded with a Bruker AVANCE III 400MHz NMR spectrometer using TMS (Tetramethyl silane) as the internal standard. The measurement was performed at $80^{\circ} \mathrm{C}$ and DMSO- $\mathrm{d}_{6}$ was used as a solvent. Fluorescence spectra of the cellulose-based fluorescent materials and epoxy coating films were measured by Hitachi F-4600 Spectrofluorometer equipped with a xenon (Xe) lamp (150W). The differential scanning calorimetry (DSC) measurements were performed on a Mettler-Toledo MET DSC under a high-purity nitrogen atmosphere with a flowing rate of $60 \mathrm{~mL} / \mathrm{min}$. Each sample $(\sim 7 \mathrm{mg})$ was sealed in an aluminum crucible and then heated from -30 to $250^{\circ} \mathrm{C}$ with heating rate of $10^{\circ} \mathrm{C} / \mathrm{min}$. Mechanical properties of epoxy coating films were carried out using a Universal Mechanical Testing Machine (Instron 5569A) with a crosshead speed of 5mm/min. Before testing, all the samples were cut into $40 \times 5 \mathrm{~mm}$ splines. The tensile properties of each sample were reported as the average of five measurements. The standard properties of epoxy coating films included hardness (GB/T6739-2006), adhesion (GB/T9286-1988), and flexibility (GB/T6742-2007).

\section{Results And Discussion}

\subsection{Fluorescence properties of cellulose-based fluorescent materials}


Corncob cellulose and MCC were firstly dissolved in $\mathrm{DMSO} / \mathrm{DBU} / \mathrm{CO}_{2}$ system and then reacted with four aromatic organics contained the luminescent groups of 2-BMN, Coumarin 151, 1-BMP and 9-CMA to form cellulose-based fluorescent materials (namely the obtained fluorescent cellulose carbonates), respectively. FTIR spectra of the corncob cellulose, MCC and the synthesized cellulose-based fluorescent materials are shown in Figure 2(a,b). From the figure, it appears the absorbance peak of $C=0$ at $\sim 1743 \mathrm{~cm}^{-1}$ in the curves of cellulose derivatives ( $A, B, C$, and $D$ ), which indicate the formation of ester groups. Furthermore, the absorption peaks of benzene ring from $1523 \mathrm{~cm}^{-1}$ to $1445 \mathrm{~cm}^{-1}$ and $725 \mathrm{~cm}^{-1}$ are also found on the FT-IR curves of these cellulose derivatives, which is different from the original corncob cellulose and MCC. The results clearly exhibit fluorescent groups of 2-BMN, Coumarin 151, 1-BMP and 9-CMA are all successfully grafted onto the molecular chains of the corncob cellulose and MCC.

${ }^{1} \mathrm{H}-\mathrm{NMR}$ spectra of synthesized cellulose-based fluorescent materials using MCC are shown in Figure 3 . The obvious presence of the introduced aromatic group and heterocyclic groups are seen in graphs. The signals between 3.15 and $5.10 \mathrm{ppm}$ are attributed to the cellulose skeleton. The degree of substitution (DS) of the cellulose-based fluorescent materials were calculated ( $\mathrm{DSA}=0.40 \otimes \mathrm{DSB}=0.32 \llbracket \mathrm{DSC}=0.28 \otimes \mathrm{DSD}=0.23$ ).

The normalized fluorescent emission spectra of the cellulose-based fluorescent materials are shown in Figure 5 (a). Under the irradiation of $365 \mathrm{~nm}$ ultraviolet (UV) light, the four kinds of cellulose-based fluorescent materials show different fluorescence response. From Figure 5(a), it is clear that the emission peaks $\left(\lambda_{\max }\right)$ of these cellulose-based fluorescent materials are separated at $424 \mathrm{~nm}(A), 470 \mathrm{~nm}$ (B), $505 \mathrm{~nm}$ (C), and $579 \mathrm{~nm}$ (D). Figure 5(b) gives the UV-vis absorption of the cellulose-based fluorescent materials. Good absorption in the range from $250 \mathrm{~nm}$ to $365 \mathrm{~nm}$ can be clearly exhibited for all the cellulose-based fluorescent materials. These cellulose-based fluorescent materials prepared by corncob cellulose show the same fluorescence response with MCC derivatives (as shown in Figure 5(c)).

\subsection{Fluorescence properties of epoxy coating films}

As shown in Figure 6(a-d), Coumarin 151 were simply mixed with cellulose and utilized to graft onto the molecular skeleton of cellulose, respectively. Both the above ways added the same mass of Coumarin 151 . And then both of them were exposed under visible and $365 \mathrm{~nm}$ UV light. It can be clearly seen that the color of the mixture of cellulose and Coumarin 151 is rather $\operatorname{dim}$ (Figure 6(c)). This result is attributed to the ACQ of Coumarin 151. After grafting Coumarin 151 on the molecular skeleton of cellulose, because of the limitation of the molecular structure, it shows that the ACQ phenomenon is obviously inhibited. As a result, the color of the Coumarin 151 grafted onto cellulose (i.e. the obtained cellulose-based fluorescent material) is much brighter under 365nm UV irradiation (Figure 6(d)).

After that, the mixture of cellulose and Coumarin 151 is introduced into epoxy resin to prepare coating material with stirring continuously for $10 \mathrm{~min}$, as exhibited in Figure 6(e). There was no obvious fluorescence response inside the coating material under UV irradiation $(365 \mathrm{~nm})$. Only weak fluorescence response appeared on the surface of the coating material. It probably owes to the serious ACQ of Coumarin 151 inside the coating material. Even small amounts without good dispersion will cause fluorescence quenching. The 
ACQ phenomenon can be also found in the coating film (see Figure 6(g-h). However, when the similar process was used to mix Coumarin 151 grafted cellulose derivative with epoxy resin, it presents a rather bright blue fluorescence under UV irradiation (see Figure 6(f)). Furthermore, the coating film with the addition of cellulosebased fluorescent material as pigment also shows rather bright blue color under UV-light (Figure 6(i-j)). That is to say, ACQ phenomenon in the coating material has been greatly suppressed. Zhang and coworker (Tian et al., 2016, 2018) had ever noticed the similar inhibition of ACQ by grafting fluorescent groups on the molecular skeleton of cellulose with the use of common ionic liquid system. The coating films obtained from cellulosebased fluorescent material showed rather good fluorescence response and bright colors. By using of the reversible $\mathrm{DMSO} / \mathrm{DBU} / \mathrm{CO}_{2}$ system in our research, cellulose-based fluorescent materials (i.e the cellulose carbonates) are successfully synthesized under relative mild reaction condition at $30-50^{\circ} \mathrm{C}$, which is lower than the reaction temperature reported by using common ionic liquid at $\sim 150^{\circ} \mathrm{C}$ (Tian et al., 2016, 2018). Then they can be prepared of epoxy-based fluorescent coating material for efficient inhibition of ACQ, which has not been clearly reported previously.

The prepared epoxy-based fluorescent coatings materials were continuously cured into coating films. The fluorescent emission spectra of the films are shown in Figure 7. Meanwhile, the epoxy resin films with pure corncob cellulose and without any additives were used as comparable samples. From Figure 7, the emission peaks of fluorescent films including the cellulose-based fluorescent materials had obvious fluorescence response with the emission peaks $\left(\lambda_{\max }\right)$ at $435 \mathrm{~nm}(A), 476 \mathrm{~nm}(B), 512 \mathrm{~nm}(C)$ and $594 \mathrm{~nm}(D)$, respectively. The $\lambda_{\max }$ is basically the same and very slightly higher than the value from the cellulose-based fluorescent materials. However, the pure epoxy resin film and the film only with corncob cellulose do not show obvious fluorescence response. These results show that the fluorescence response and the color under $365 \mathrm{~nm}$ UV light of the epoxy-based coating films are actually decided by the added cellulose-based fluorescent materials. Therefore, it is feasible to use the obtained cellulose-based fluorescent materials to prepare epoxy-based fluorescent coating materials and films.

\subsection{Standard, mechanical and thermal properties of epoxy coating films}

The standard properties of epoxy coating films are shown in Table 1. In comparison with pure epoxy film, the addition with cellulose and its fluorescent derivatives induces the increase of the hardness level from $\mathrm{H}$ to $2 \mathrm{H}$. Meanwhile, the increase of hardness does not impact the flexibility of the coating films. The only decrease exists on the adhesion level between coating films and metal substrate, which is from 0 to 1 . However, the result of "level 1" is still satisfied with the performance standard of national standard (GB/T9286-1988) as a high-quality coating. Furthermore, all the coating films show good water and acetone resistances. The addition of cellulose and its fluorescent derivatives does not change the stability of epoxy-based coating films.

Table 1. Standard properties of coating films prepared by epoxy with or without cellulose and its fluorescent derivatives. 


\begin{tabular}{|llllll|}
\hline Sample & Hardness & Adhesion/level & Flexibility/mm & $\begin{array}{l}\text { Water } \\
\text { resistance } \\
\left(25^{\circ} \mathrm{C}, 240 \mathrm{~h}\right)\end{array}$ & $\begin{array}{l}\text { Acetone resistance } \\
\text { (wiping method)/time }\end{array}$ \\
\hline Epoxy/A & $2 \mathrm{H}$ & 1 & 1 & Normal & $>500$ \\
\hline Epoxy/B & $2 \mathrm{H}$ & 1 & 1 & Normal & $>500$ \\
\hline Epoxy/C & $2 \mathrm{H}$ & 1 & 1 & Normal & $>500$ \\
\hline Epoxy/D & $2 \mathrm{H}$ & 1 & 1 & Normal & $>500$ \\
\hline Epoxy/Cellulose & $2 \mathrm{H}$ & 1 & 1 & Normal & $>500$ \\
\hline Epoxy & $\mathrm{H}$ & 0 & 1 & Normal & $>500$ \\
\hline
\end{tabular}

Table 2. Mechanical properties of epoxy coating films with or without cellulose and its fluorescent derivatives.

\begin{tabular}{|llll|}
\hline Sample & $\begin{array}{l}\text { Tensile strength } \\
(\mathrm{MPa})\end{array}$ & $\begin{array}{l}\text { Tensile modulus } \\
(\mathrm{MPa})\end{array}$ & $\begin{array}{l}\text { Elongation at break } \\
(\%)\end{array}$ \\
\hline Epoxy/A & $78.17 \pm 1.53$ & $35.44 \pm 1.60$ & $2.48 \pm 0.01$ \\
\hline Epoxy/B & $75.03 \pm 2.28$ & $31.97 \pm 0.54$ & $2.78 \pm 0.05$ \\
\hline Epoxy/C & $75.13 \pm 2.29$ & $36.11 \pm 0.88$ & $2.56 \pm 0.03$ \\
\hline Epoxy/D & $83.21 \pm 0.67$ & $36.69 \pm 1.20$ & $2.60 \pm 0.02$ \\
\hline Epoxy/Cellulose & $75.73 \pm 1.66$ & $35.85 \pm 1.52$ & $2.46 \pm 0.05$ \\
\hline Epoxy & $55.63 \pm 0.85$ & $19.21 \pm 0.78$ & $4.30 \pm 0.02$ \\
\hline
\end{tabular}

Mechanical properties of epoxy coating films are investigated by the tensile test. The tensile curves of the coating films are shown in Figure 8 and the mechanical properties are listed in Table 2. After the addition of cellulose and its fluorescent derivatives into coating films, the tensile curves show greatly change (see Figure 6). Tensile strength and tensile modulus are respectively increase from $55.63 \pm 0.85 \mathrm{MPa}$ to $75-83 \mathrm{MPa}$ and from 19.21 $\pm 0.78 \mathrm{MPa}$ to $31-35 \mathrm{MPa}$ (see Table 2). The increase of tensile strength and tensile modulus reaches $\sim 39 \%$ and $\sim 66 \%$. Although, at the same time, the elongation of the coating films decreases from $4.30 \pm 0.02 \%$ to $2.46-2.78 \%$, it does not reflect obvious change in the essential stiffness and toughness. That is because cellulose and its fluorescent derivatives are rigid additives as to epoxy resin. The addition of cellulose and its fluorescent derivatives increase hardness and rigidity of epoxy coating films. It increases the mechanical properties to some extent. That is consistent with previous research findings that cellulose has ability to improve the mechanical property of epoxy matrix (Alamri \& Low, 2013; Canche-Escamilla et al., 2002; HerreraFranco \& AguilarVega, 1997; Jang et al., 2012; Masoodi et al., 2012; Sdrobis et al., 2012).

DSC measurement is selected to determine the glass transition temperature $\left(T_{g}\right)$ of all the epoxy coating films. The DSC heating curves are shown in Figure 9 . The $T_{g}$ of pure epoxy coating film was $90.0^{\circ} \mathrm{C}$. After added cellulose and its fluorescent derivatives, the $T_{g}$ of coating films slightly decrease to range from $88.4^{\circ} \mathrm{C}$ to 
$89.6^{\circ} \mathrm{C}$. The use of cellulose-based fluorescent materials does not change the thermal property of the coating films.

\section{Conclusion}

Cellulose-based fluorescent materials were successfully achieved by use of reversible DMSO/DBU/CO 2 system under relative mild conditions. The obtained cellulose-based fluorescent materials show good fluorescence response and bright colors under 365nm UV light. Due to the limitation of the molecular structure, the intrinsic ACQ phenomenon of conventional organic fluorescent pigments can be inhabited. Then the cellulose-based fluorescent materials are introduced into epoxy resin to prepare fluorescent coating. It also shows rather good fluorescence response and the bright color. Moreover, the addition of cellulose derivatives increases the mechanical properties of the coating film. Solvent resistance and thermal property of the coating films generally remain unchanged.

\section{Declarations}

\section{Funding info}

The National Natural Science Foundation of China (21978310 \& 51773217), the Key Research and Development Program of Shandong Province (2019JZZY020217), Youth Innovation Promotion Association CAS (2017339), Ningbo Natural Science Foundation (2019A610026).

\section{Competing interests}

There is no competing interest to declare.

\section{Data Availability}

All data generated or analyzed during this study are included in this published article.

\section{Author contribution}

Qinghua Cao carried out the majority parts of experiments such as dissolving and modification of cellulose. He also carried out the majority parts of analysis of experimental results and writing of manuscript.

Jinyue Dai guided and worked together on the preparation and characterization of epoxy coating film.

Xin Bao helped performing the analysis and gave constructive discussion during experiment.

Zhenyu Zhang helped to carry out the analysis of experiment and some part of manuscript preparation.

Fei Liu helped performing the analysis of partially experimental data.

Yuhong Feng guided the research methodology of this manuscript.

Haining Na guided the analysis of partially experimental data and the writing of this manuscript. 
Jin Zhu guided the general conception of this study.

\section{Animal Research (Ethics)}

Not applicable.

\section{Ethics approval}

Not applicable.

\section{Consent to participate (Ethics)}

Not applicable.

\section{Consent to publish (Ethics)}

Not applicable.

\section{Acknowledgement}

We are grateful for the financial support by the National Natural Science Foundation of China (21978310 \& 51773217), the Key Research and Development Program of Shandong Province (2019JZZY020217), Youth Innovation Promotion Association CAS (2017339), Ningbo Natural Science Foundation (2019A610026).

\section{References}

Alamri, H., \& Low, I. M. (2013). Effect of water absorption on the mechanical properties of nanoclay filled recycled cellulose fibre reinforced epoxy hybrid nanocomposites. Composites Part A-Applied Science And Manufacturing, 44, 23-31.

Andanson, J.-M., Bordes, E., Devemy, J., Leroux, F., Padua, A. A. H., \& Gomes, M. F. C. (2014). Understanding the role of co-solvents in the dissolution of cellulose in ionic liquids. Green Chemistry, 16(5), 2528-2538.

Birks, J. B. (1971). Excited states. (Book reviews: Photophysics of aromatic molecules). Science, $174,580$.

Canche-Escamilla, G., Rodriguez-Laviada, J., Cauich-Cupul, J. I., Mendizabal, E., Puig, J. E., \& Herrera-Franco, P. J. (2002). Flexural, impact and compressive properties of a rigid-thermoplastic matrix/cellulose fiber reinforced composites. Composites Part A-Applied Science And Manufacturing, 33(4), 539-549.

Druel, L., Niemeyer, P., Milow, B., \& Budtova, T. (2018). Rheology of cellulose- [DBNH][CO 2 Et] solutions and shaping into aerogel beads. Green Chemistry, 20(17), 3993-4002.

Fink, H. P., Weigel, P., Purz, H. J., \& Ganster, J. (2001). Structure formation of regenerated cellulose materials from NMMO-solutions. Progress In Polymer Science, 26(9), 1473-1524.

Fischer, S., Leipner, H., Thümmler, K., Brendler, E., \& Peters, J. (2003). Inorganic molten salts as solvents for cellulose. Cellulose, 10(3), 227-236. 
Heinze, T., \& Liebert, T. (2001). Unconventional methods in cellulose functionalization. Progress In Polymer Science, 26(9), 1689-1762.

HerreraFranco, P. J., \& AguilarVega, M. D. (1997). Effect of fiber treatment on the mechanical properties of LDPE-henequen cellulosic fiber composites. Journal Of Applied Polymer Science, 65(1), 197-207.

Jang, J. Y., Jeong, T. K., Oh, H. J., Youn, J. R., \& Song, Y. S. (2012). Thermal stability and flammability of coconut fiber reinforced poly(lactic acid) composites. Composites Part B-Engineering, 43(5), 2434-2438.

Klemm, D., Heublein, B., Fink, H. P., \& Bohn, A. (2005). Cellulose: Fascinating biopolymer and sustainable raw material. Angewandte Chemie-International Edition, 44(22), 3358-3393.

Koehler, S., \& Heinze, T. (2007). New solvents for cellulose: Dimethyl sulfoxide/ammonium fluorides. Macromolecular Bioscience, 7(3), 307-314.

Llevot, A., Dannecker, P. K., von Czapiewski, M., Over, L. C., Soyler, Z., \& Meier, M. A. R. (2016). Renewability is not enough: Recent advances in the sustainable synthesis of biomass-derived monomers and polymers.

Chemistry-a European Journal, 22(33), 11509-11520.

Masoodi, R., El-Hajjar, R. F., Pillai, K. M., \& Sabo, R. (2012). Mechanical characterization of cellulose nanofiber and bio-based epoxy composite. Materials \& Design, 36, 570-576.

Mccormick, C. L., \& Dawsey, T. R. (1990). Preparation of cellulose derivatives via ring-opening reactions with cyclic reagents in lithium chloride/N,N-dimethylacetamide. Macromolecules, 23(15), 3606-3610.

Onwukamike, K. N., Tassaing, T., Grelier, S., Grau, E., Cramail, H., \& Meier, M. A. R. (2017). Detailed understanding of the $\mathrm{DBU} / \mathrm{CO}_{2}$ switchable solvent system for cellulose solubilization and derivatization. Acs Sustainable Chemistry \& Engineering, 6(1), 1496-1503.

Rahman, M. H., Liao, S.-C., Chen, H.-L., Chen, J.-H., Ivanov, V. A., Chu, P. P. J., \& Chen, S.-A. (2009). Aggregation of conjugated polymers in aromatic solvent. Langmuir, 25(3), 1667-1674.

Sdrobis, A., Darie, R. N., Totolin, M., Cazacu, G., \& Vasile, C. (2012). Low density polyethylene composites containing cellulose pulp fibers. Composites Part B-Engineering, 43(4), 1873-1880.

Song, L., Yang, Y., Xie, H., \& Liu, E. (2015). Cellulose Dissolution and inSitu grafting in a reversible system using an organocatalyst and carbon dioxide. Chemsuschem, 8(19), 3217-3221.

Teng, J., Zhang, Y., Ruan, X. (2010). Some improtant sciemtific problems for development of renewable and new energy - The only way for development of non-fossil energy. Progress in Geophysiscs, 25(4), 1115-1152.

Tian, W., Zhang, J., Yu, J., Wu, J., Nawaz, H., Zhang, J., He, J., \& Wang, F. (2016). Cellulose-based solid fluorescent materials. Advanced Optical Materials, 4(12), 2044-2050.

Tian, W., Zhang, J., Yu, J., Wu, J., Zhang, J., He, J., \& Wang, F. (2018). Phototunable full-color emission of cellulose-based dynamic fluorescent materials. Advanced Functional Materials, 28(9), 1703548. 
Wu, J., Zhang, J., Zhang, H., He, J. S., Ren, Q., \& Guo, M. (2004). Homogeneous acetylation of cellulose in a new ionic liquid. Biomacromolecules, 5(2), 266-268.

Xie, H., Yu, X., Yang, Y., \& Zhao, Z. K. (2014). Capturing $\mathrm{CO}_{2}$ for cellulose dissolution. Green Chemistry, 16(5), 2422-2427.

Xu, Q., Song, L., Zhang, L., Hu, G., Du, J., Liu, E., Zheng, Q., Liu, Y., Li, N., \& Xie, H. (2017). Organocatalytic cellulose dissolution and in situ grafting of epsilon-caprolactone via ROP in a reversible $\mathrm{DBU} / \mathrm{DMSO} / \mathrm{CO}_{2}$ system. Chemistryselect, 2(24), 7128-7134.

Yang, Y., Song, L., Peng, C., Liu, E., \& Xie, H. (2015). Activating cellulose via its reversible reaction with $\mathrm{CO}_{2}$ in the presence of 1,8-diazabicyclo [5.4.0] -undec-7-ene for the efficient synthesis of cellulose acetate. Green Chemistry, 17(5), 2758-2763.

Zhang, H., Wu, J., Zhang, J., \& He, J. S. (2005). 1-Allyl-3-methylimidazolium chloride room temperature ionic liquid: A new and powerful nonderivatizing solvent for cellulose. Macromolecules, 38(20), 8272-8277.

Zhang, L., Shi, W., Wang, J., Jin, L., Hu, G., Zheng, Q., Xie, H., \& Chen, P. (2019). Unique gelation and rheological properties of the cellulose/ $\mathrm{CO}_{2}$-based reversible ionic liquid/DMSO solutions. Carbohydrate Polymers, 222, 115024.

Zhang, Q., Oztekin, N. S., Barrault, J., Vigier, K. D. O., \& Jerome, F. (2013). Activation of microcrystalline cellulose in a $\mathrm{CO}_{2}$-Based switchable system. Chemsuschem, 6(4), 593-596.

Zheng, Y., Shao, Z., Li, Y., Wang, W. (2005). Ether-ester derivatives of cellulose and their applications. Journal of Cellulose Science and Technology, 13(1), 61-65.

Zhou, J., \& Zhang, L. (2000). Solubility of cellulose in $\mathrm{NaOH} /$ urea aqueous solution. Polymer Journal, 32(10), 866-870.

\section{Figures}



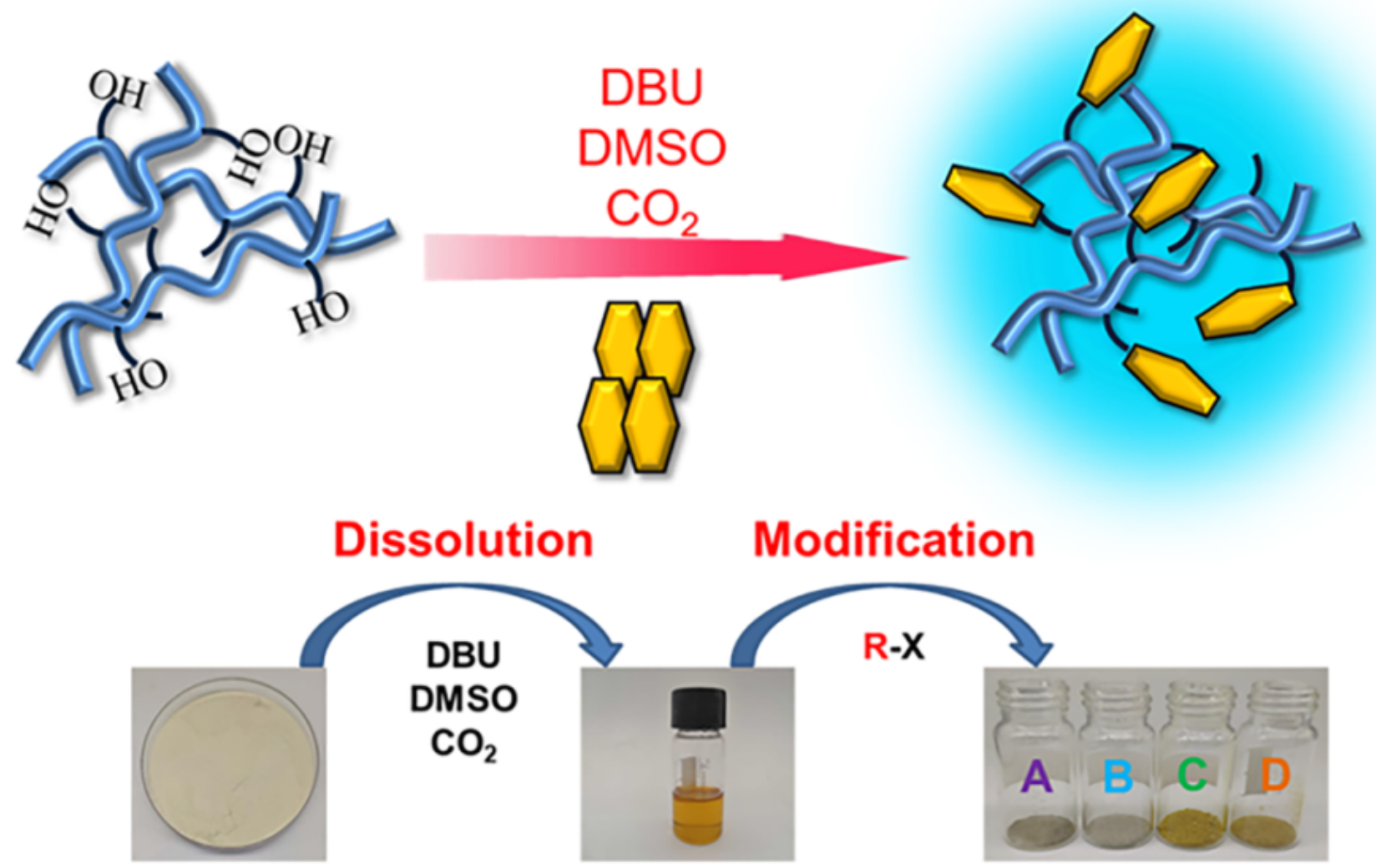

Cellulose

Cellulose-based fluorescent material

R: $A$ 1-BMN<smiles>[B]Nc1ccc2c(C(F)(F)F)cc(=O)oc2c1</smiles>
Coumarin 151<smiles>BrCc1ccc2ccc3cccc4ccc1c2c34</smiles>

1-BMP<smiles></smiles>

CMA

Figure 1

Synthesis of cellulose-based fluorescent materials. 

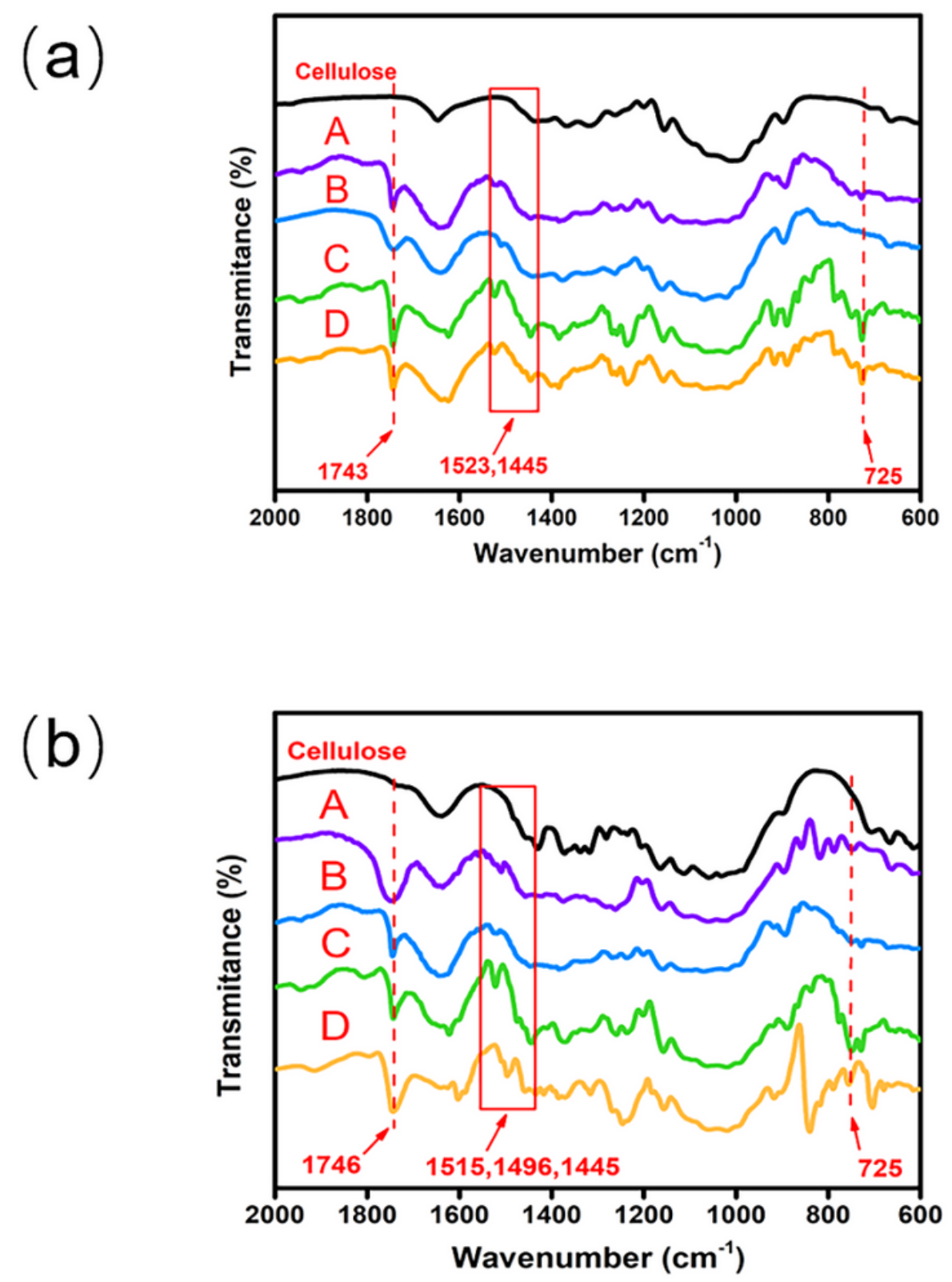

\section{Figure 2}

(a) FT-IR spectra of corncob cellulose and synthesized cellulose-based fluorescent materials grafted with A (2BMN), B (Coumarin 151), C (1-BMP), D (9-CMA); (b) FT-IR spectra of MCC and synthesized cellulose-based fluorescent materials grafted with A (2-BMN), B (Coumarin 151), C (1-BMP), D (9-CMA). 

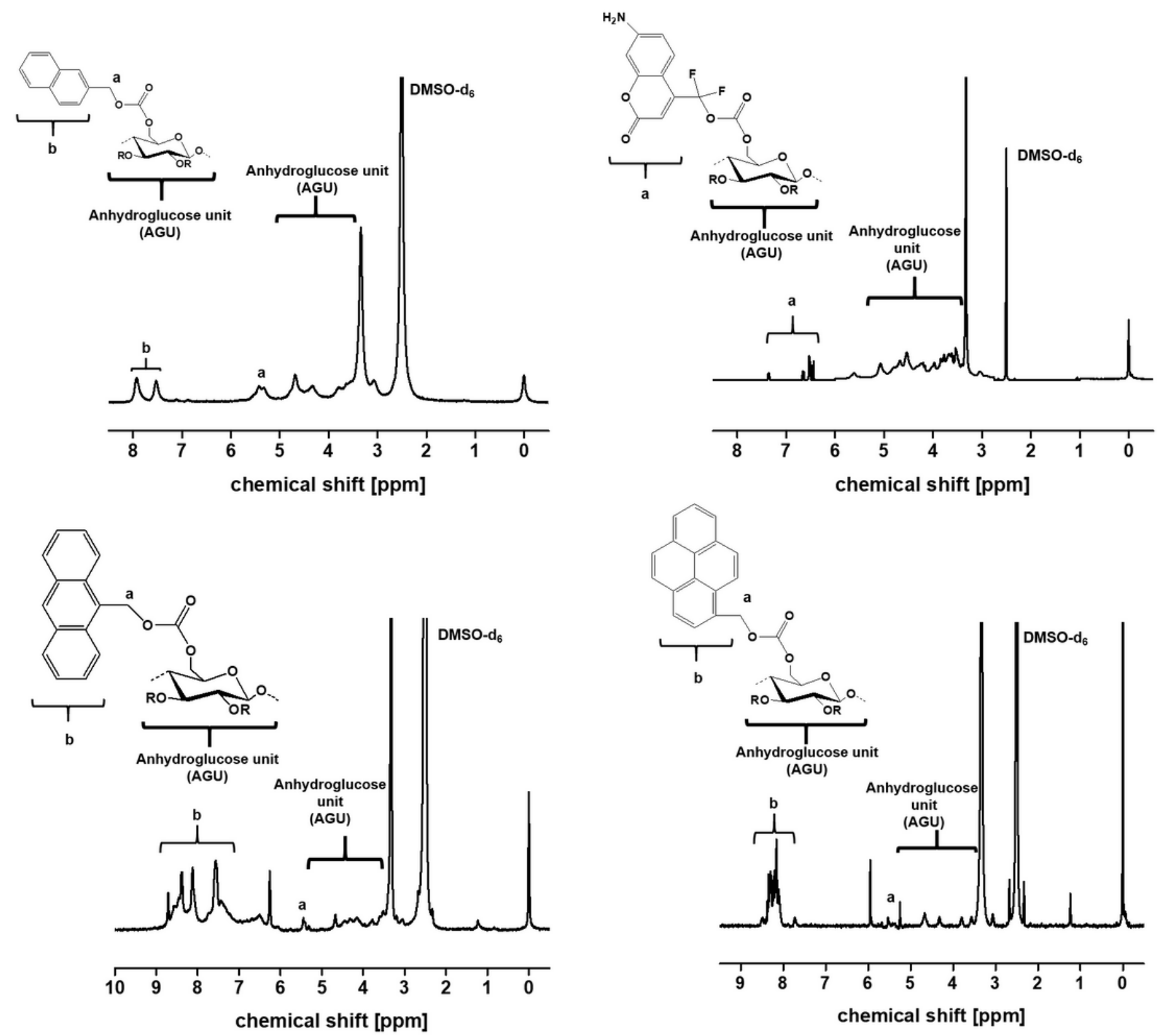

Figure 3

Color of cellulose-based fluorescent materials prepared by MCC under visible light and its fluorescent images of them under $365 \mathrm{~nm}$ UV light. 


\section{Visible light UV light $365 \mathrm{~nm}$}

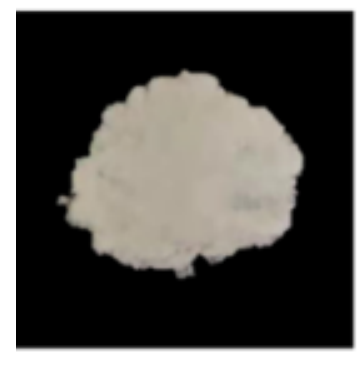

MCC
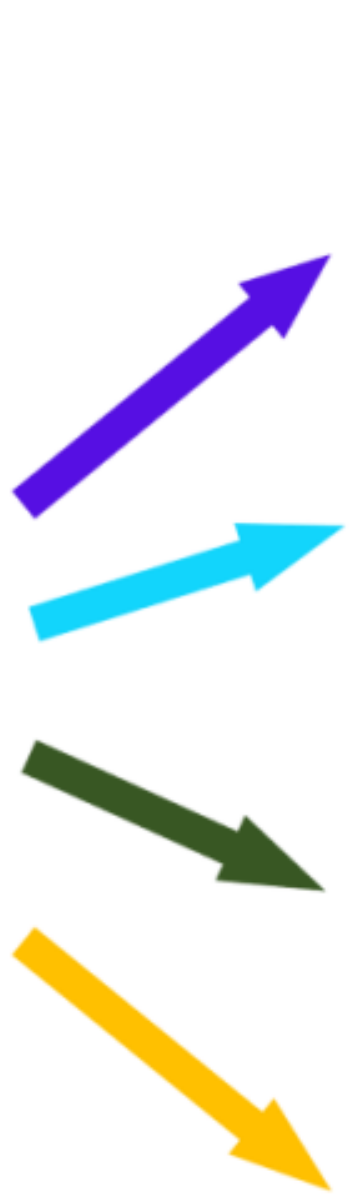

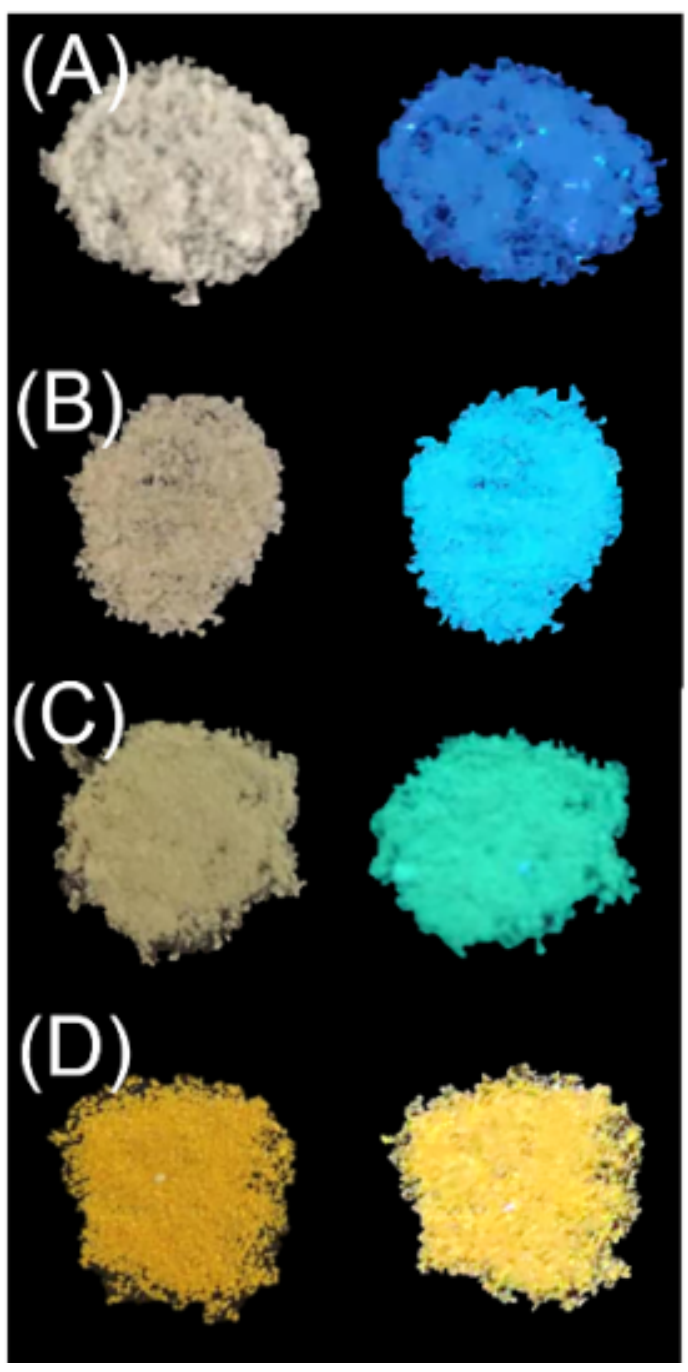

Figure 4

Color of cellulose-based fluorescent materials prepared by MCC under visible light and its fluorescent images of them under 365nm UV light. 
(a)



(b)
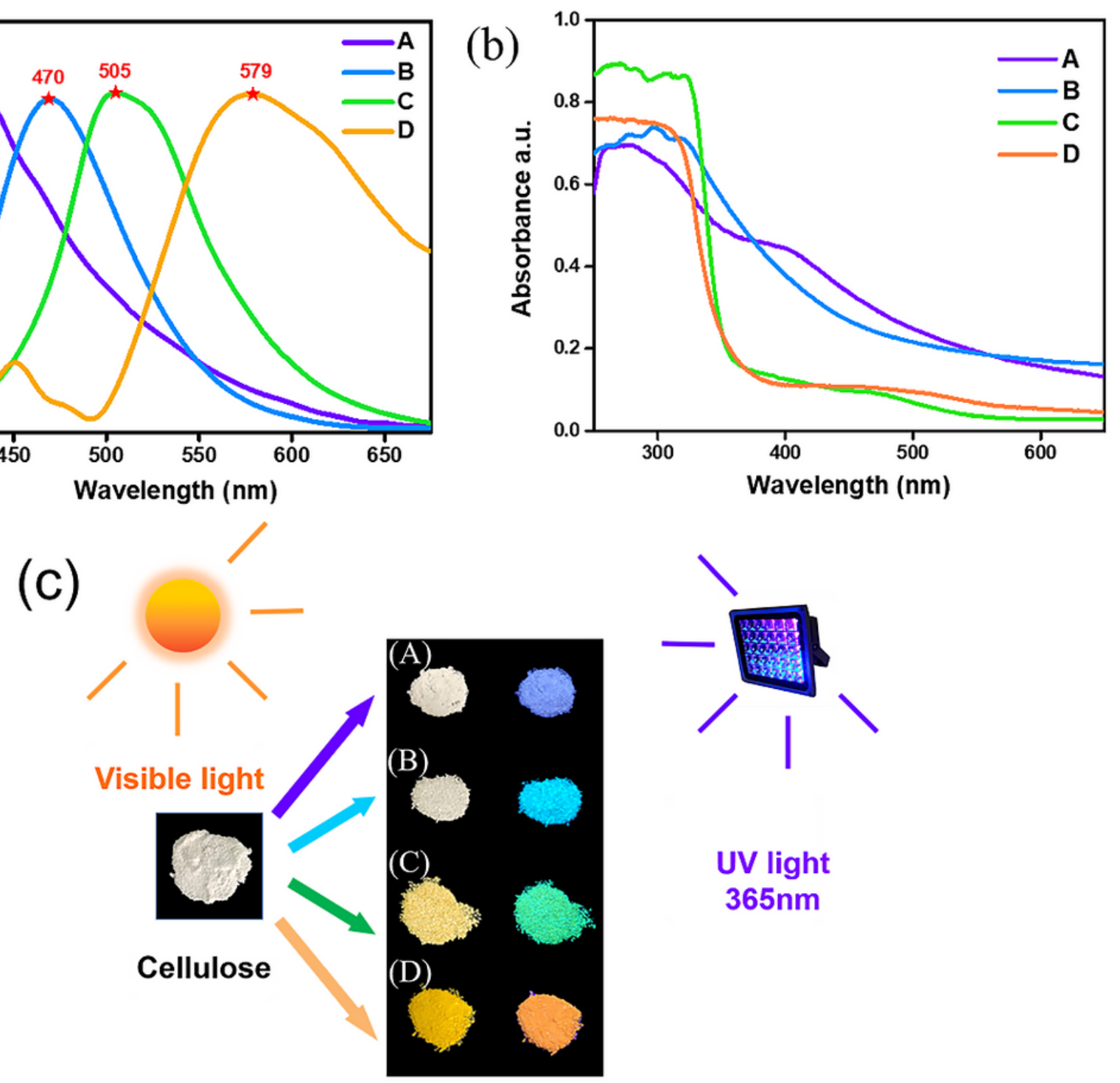

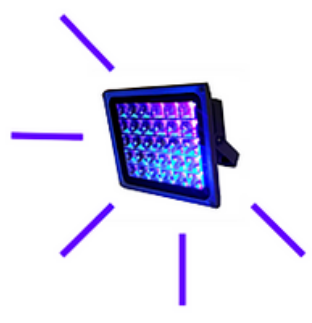

UV light

$365 \mathrm{~nm}$

Figure 5

(a) Emission spectra and (b) UV-vis absorption spectra of cellulose-based fluorescent materials grafted with A (2-BMN), B (Coumarin 151), C (1-BMP) and D (9-CMA) with 365nm exciting light; (c) Color of cellulose-based fluorescent materials under visible light and its fluorescent images of them under $365 \mathrm{~nm}$ UV light. 
(cellulose mixed with

luminogen powder)

\section{Visible light}

\author{
UV light
}

$365 \mathrm{~nm}$

\section{UV light
$365 \mathrm{~nm}$ \\ UV light
$365 \mathrm{~nm}$}

\section{Mixed}

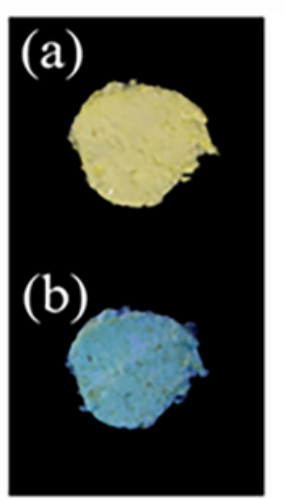

Grafted (luminogen powder Grafted onto cellulose)

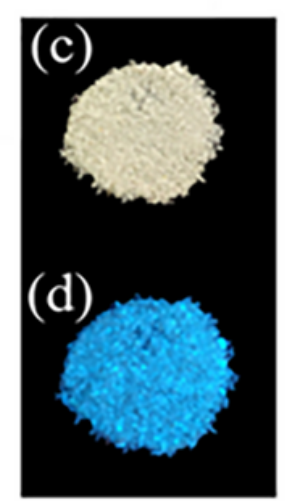

Added to epoxy resin as an additive
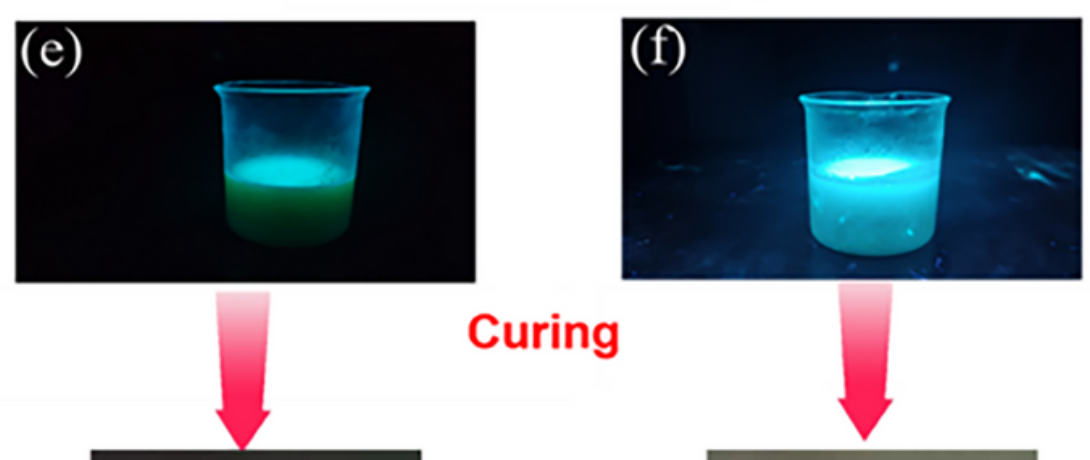

Curing

\section{Visible light}

\section{UV light $365 \mathrm{~nm}$}
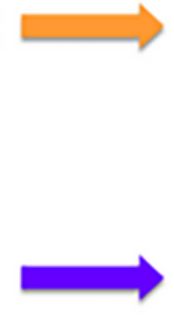

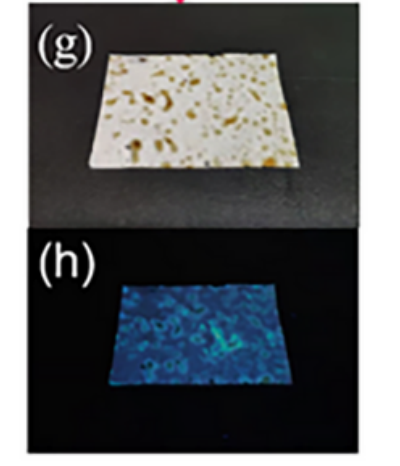

None uniform fluorescence

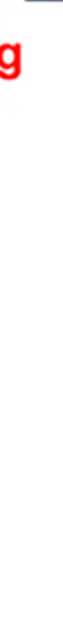

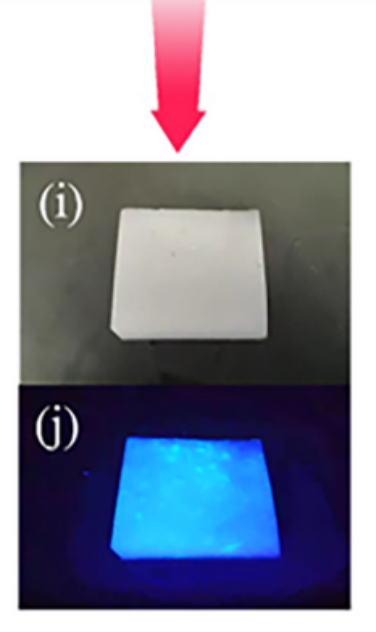

Uniform fluorescence

\section{Figure 6}

The anchoring and diluting effect of cellulose skeleton efficiently inhibited the ACQ of luminogens. The cellulose mixed with Coumarin 151 powder under (a) visible light and (b) 365nm UV-light; The Coumarin 151 grafted onto cellulose skeleton under (c) visible light and (d) 365nm UV-light. Epoxy added with mixture (e) and (f) cellulose-based fluorescent material. Epoxy coating film added with the mixture of cellulose and Coumarin 151 powder under (g) visible light and (h) 365nm UV-light. Epoxy coating film added with cellulosebased fluorescent material under (i) visible light and (j) 365nm UV-light. 


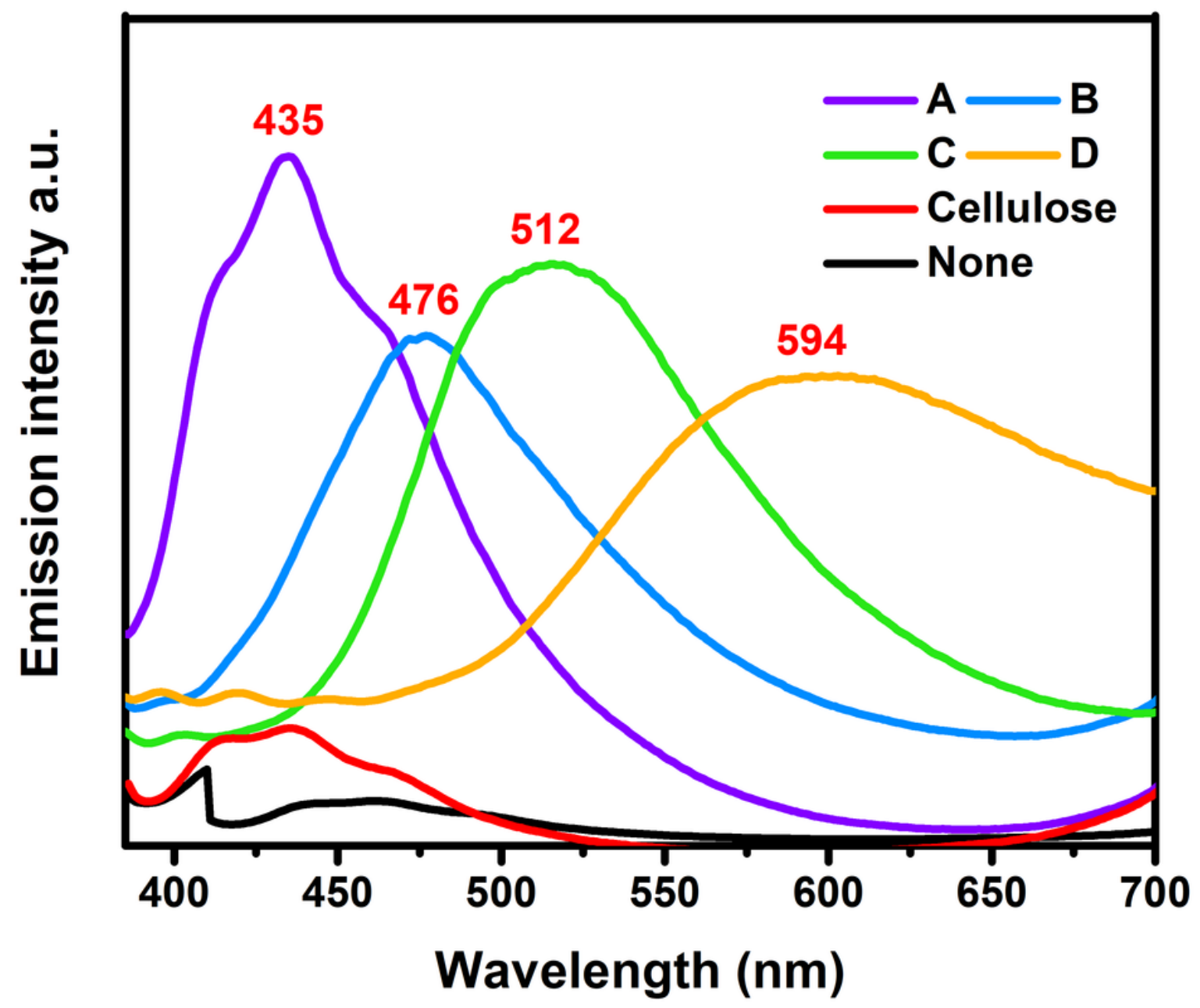

Figure 7

Emission spectra of epoxy coating films with or without cellulose and cellulose-based fluorescent materials grafted with A (2-BMN), B (Coumarin 151), C (1-BMP) and D (9-CMA) under 365nm UV light. 


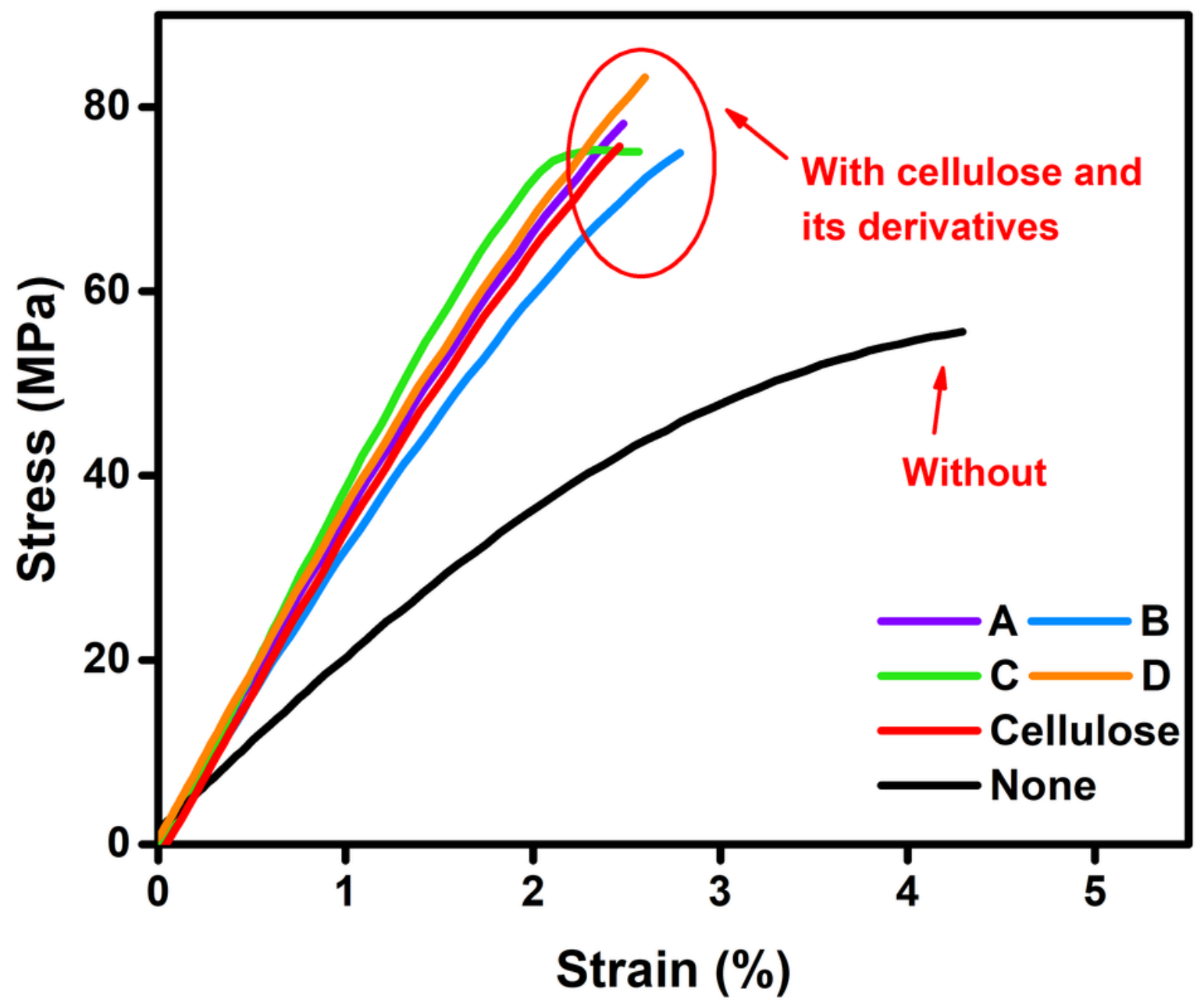

Figure 8

Tensile curves for epoxy coating films with or without cellulose and its fluorescent derivatives grafted with $A$ (2-BMN), B (Coumarin 151), C (1-BMP) and D (9-CMA). 


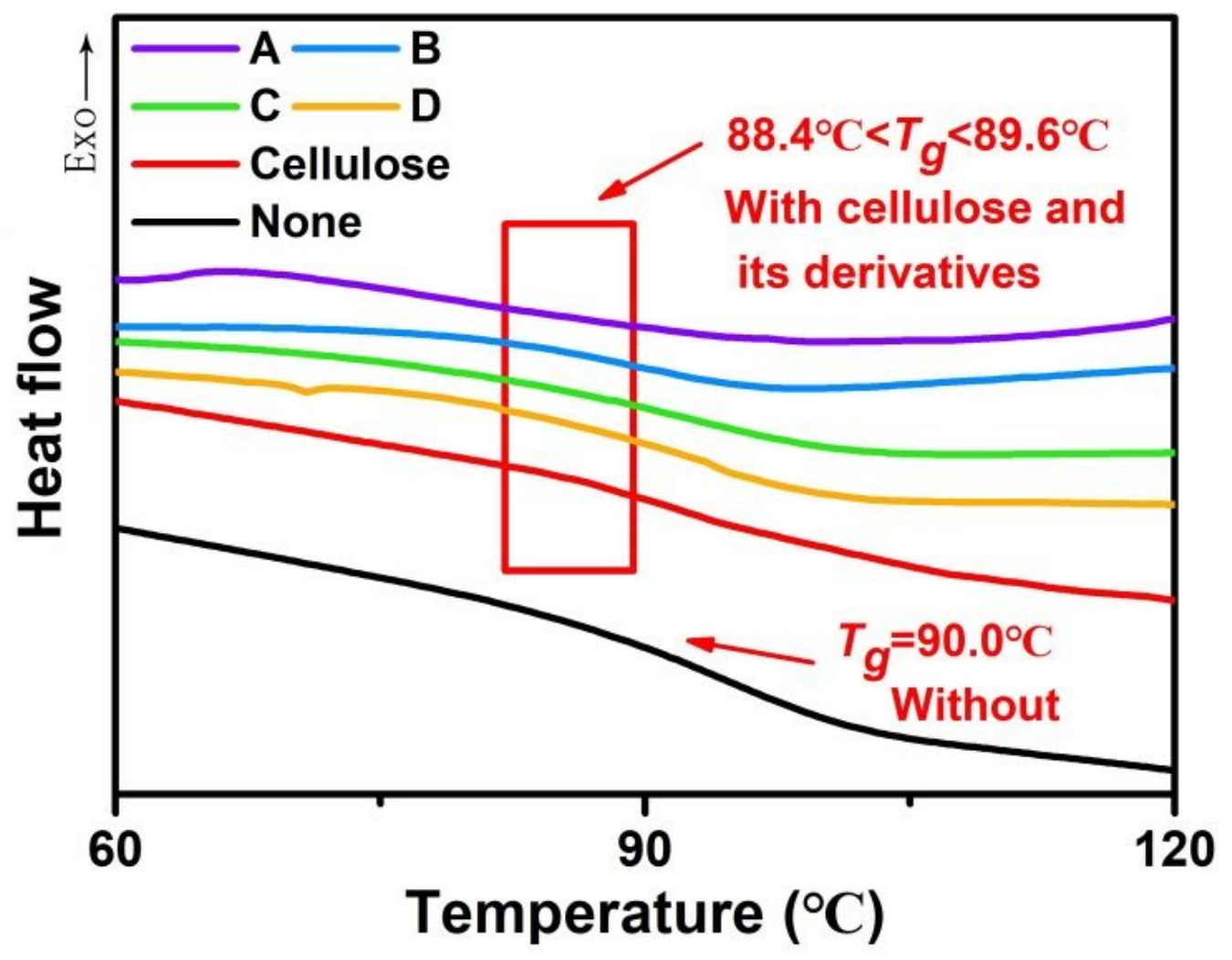

Figure 9

DSC heating curves for epoxy coating films with or without cellulose and its fluorescent derivatives grafted with A (2-BMN), B (Coumarin 151), C (1-BMP) and D (9-CMA). 